Ann. Zootech., I973, $22(2)$, I57-I65.

\title{
UTILISATION DU MAÏS « OPAQUE 2 » PAR LE PORC EN CROISSANCE-FINITION
}

\author{
D. BOURDON, Y. HENRY et D. DURAND (1) \\ avec la collaboration technique de B. Dabiel, G. Consel, et M. Caron \\ Station de Recherches sur l'Élevage des Porcs, \\ Centre national de Recherches zootechniques, I. N.R. A., \\ 78350 Jouy en Josas
}

\section{RÉSUMÉ}

Une expérience réalisée, sur 40 porcs de race Large White, entre 29 et $90 \mathrm{~kg}$ de poids vif, a eu pour but d'étudier les conditions d'utilisation du maïs $O$ paque 2 en comparaison avec le maïs normal, dans des régimes complémentés par du tourteau de soja.

Quatre lots de ro animaux sont constitués et reçoivent, selon le mode semi-ad libitum, les régimes suivants :

- Lot I : Régime témoin maïs normal-tourteau de soja $(\mathrm{I} 6,5$ p. Ioo de matières azotées et o,8 p. Ioo de lysine en croissance ; 14,5 p. Ioo de matières azotées et o,65 p. Ioo de lysine en finition).

- Lot 2 : Régime maïs Opaque 2-tourteau de soja, à même niveau d'apport de matières azotées et de lysine que le régime témoin.

- Lot 3: Régime maîs Opaque 2 à taux réduit de soja (I 4,5 p. roo de matières azotées en croissance et I 2,5 en finition), supplémenté en lysine.

- Lot 4: Régime 3 rééquilibré au même taux de matières azotées que le régime témoin (lot $\mathrm{I}$ ), avec un apport complémentaire de gluten de maïs.

Sur l'ensemble de l'expérience, les performances obtenues sont sensiblement les mêmes pour les deux types de maïs, à l'exception toutefois du lot 3 , à taux azoté réduit, qui se révèle infér:eur aux trois autres. Par ailleurs, durant la première phase d'engraissement, les animaux soumis aux régimes à base de maïs $O p a q u e ~ 2$ réalisent une croissance légèrement inféricure à celle des témoins.

\section{INTRODUCTION}

Les nombreux travaux consacrés, au cours de ces dernières années, à l'utilisation de mais hybrides Opaque 2, riches en lysine, dans l'alimentation du porc en croissance, ont permis de conclure à la possibilité de réduire d'une façon notable le taux d'incorpo-

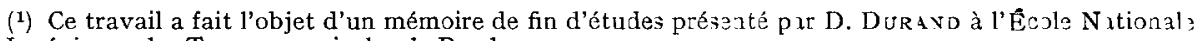
des Ingénieurs des Travaux agricoles de Bordeaux. 
ration des compléments azotés nécessaires dans les régimes à base de céréales. D'une manière générale, il a été observé qu'en régime isoazoté, à un taux suboptimum pour la croissance, le maïs $O p a q u e ~ 2$ se révèle supérieur au maïs normal, tandis qu'il procure des performances identiques à un taux de matières azotées abaissé de $2 \mathrm{p}$. Ioo environ (de I6 à I4 p. Ioo par exemple). L'économie de complément azoté peut ainsi être chiffrée à 5 ou 6 p. Ioo d'après la plupart des auteurs (Cromwer, et al., I967 ; DrEws et al., I969 ; Gipp et al., I968 ; Gallo et al., I969 ; Sinombing et al., r969). Il convient cependant de remarquer qu'au cours de ces travaux, le plus souvent de courte durée, les observations ont porté d'une manière quasi exclusive sur la vitesse de croissance et l'indice de consommation et rarement sur les caractéristiques de composition corporelle qui, cependant, sont susceptibles d'être plus ou moins fortement influencées par une diminution du taux azoté de la ration, consécutive à une supplémentation par une source azotée riche en lysine.

C'est pourquoi, après avoir retrouvé, dans un premier essai (HENRY et BouRDON, I97I), l'effet bénéfique du supplément de lysine apporté par le maīs Opaque 2 sur la rétention azotée chez le porc en croissance, nous avons entrepris une nouvelle expérience destinée à évaluer l'économie de soja qui pourrait résulter du remplacement du maïs ordinaire par l'hybride Opaque 2 , à taux de lysine et de matières azotées identiques, lorsque l'on prend en considération les performances de croissance et la composition corporelle au poids commercial d'abattage $(90 \mathrm{~kg}$ ) ou à un stade intermédiaire de la croissance $(60 \mathrm{~kg})$.

\section{I. - MATÉRIEL ET MÉTHODES}

Quarante porcelets de race Large White, d'un poids moyen initial de $28,9 \mathrm{~kg}$ et d'un âge moyen de 88 jours, sont répartis en 4 lots de Io (5 mâles castrés et 5 femelles), selon le dispositif en blocs complets d'après l'âge et le poids vif. Les régimes correspondant à ces 4 lots sont décrits dans le tableau I. La composition en acides aminés des protéines de maïs est donnée dans le tableau 2.

- Le lot $\mathrm{r}$, reçoit un régime témoin à base de maïs normal (I.N.R.A.310) et de tourteau de soja, équilibré en matières azotées et en lysine. Les pourcentages respectifs de matières azotées et de lysine sont les suivants : 16,5 et 0,80 pendant la période de croissance $(30-60 \mathrm{~kg}$ de poids vif), 14,5 et 0,65 pendant la phase de finition $(60-90 \mathrm{~kg})$.

- Dans le lot 2, le maïs normal est remplacé par un mais hybride $O$ paque 2 , ayant subi les mêmes conditions culturales. Le régime renferme des taux de matières azotées et de lysine identiques à ceux du lot I : Ia diminution du taux d'incorporation de tourteau de soja, grâce à une teneur plus élevée en lysine du maïs Opaque 2 (tabl. 2), est compensée par un apport complémentaire de matières azotées sous forme de gluten de maïs.

- Le régime 3 , également à base de maïs opaque 2 , contient un taux réduit de tourteau de soja, donc de matières azotées, mais bénéficie d'un apport supplémentaire de L-lysine sous forme libre, puisque le tryptophane, acide aminé limitant secondaire des régimes maïs-soja, est présent en plus grande quantité dans l'hybride Opaque 2 que dans l'hybride normal (généralement $+30 \mathrm{p}$. IOo). Le taux réduit de tourteau de soja a été calculé de manière à vérifier la possibilité d'une supplémentation en lysine et réaliser ainsi une économie supplémentaire de soja.

- Le régime 4, qui renferme les mêmes taux de soja et de lysine que le précédent (lot 3). est complété par une source azotée pauvre en lysine (gluten de maiis), pour obtenir une teneur en matières azotées identique à celles des lots $x$ et 2 , et contrôler ainsi l'effet de l'apport global de matières azotées, indépendamment de celui de la lysine.

Les animaux sont élevés en loges collectives, munies chacune d'un dispositif d'alimentation individuelle et d'un abreuvoir automatique. Ils sont nourris suivant le mode semi-ad libitum, à raison de deux repas longs ( $\mathrm{r}$ heure) par jour. Les régimes sont distribués sous forme de soupe ( 2 parties d'eau pour une partie d'aliment). Le contrôle de la croissance à lieu à intervalles réguliers 
UTILISATION DU MAÏS " OPAQUE 2 " PAR LE PORC

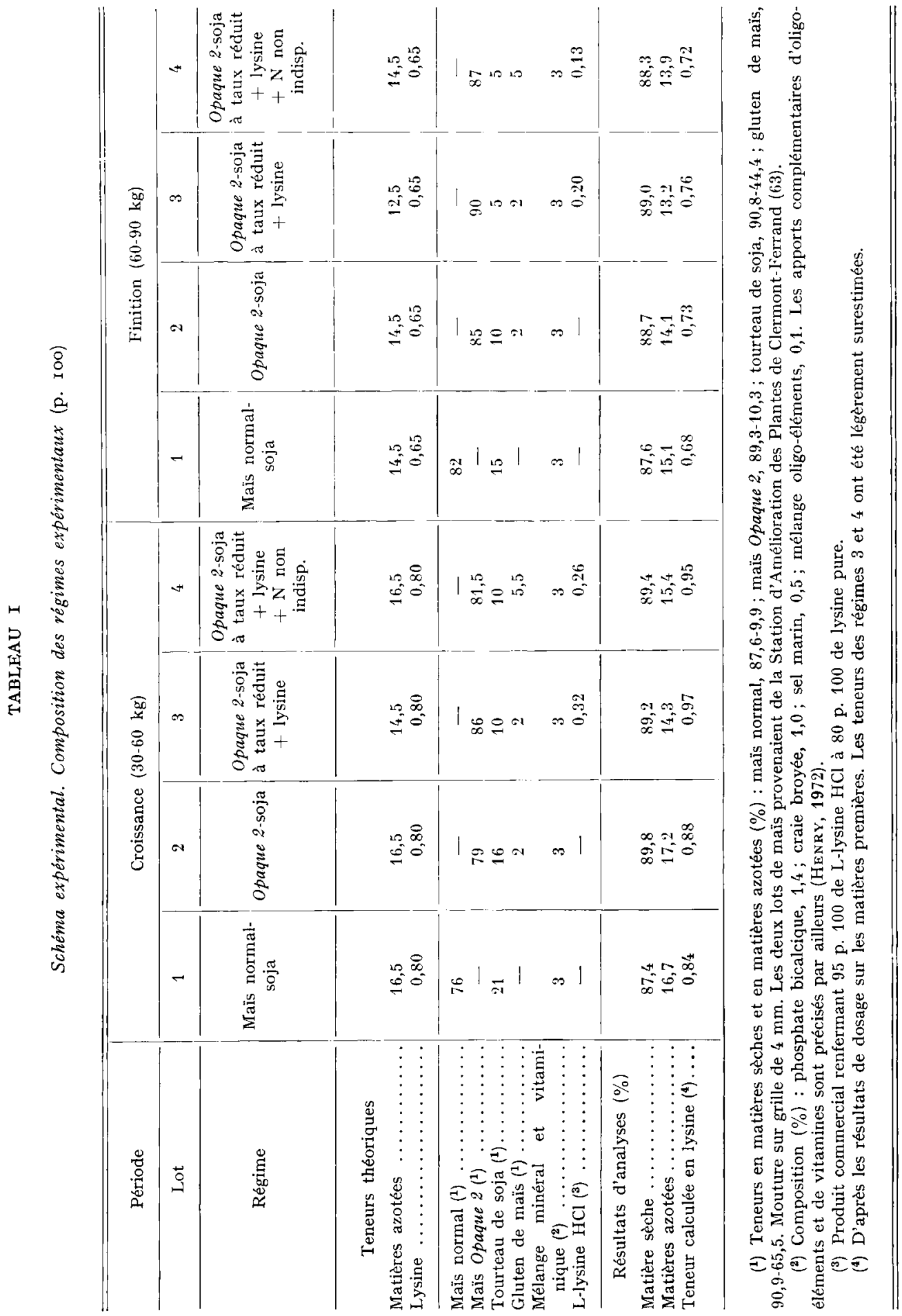


TABLEAU 2

Composition des mais en acides aminés ( $\mathrm{g} / \mathrm{I} 6 \mathrm{~g} \mathrm{~N}$ )

\begin{tabular}{|c|c|c|c|c|c|}
\hline Acide aminé & Normal & Opaque 2 & Acide aminé & Normal & Opaque 2 \\
\hline Acide aspartique .... & 6,75 & 9,95 & Valine $\ldots \ldots \ldots \ldots$ & 5,25 & 5,25 \\
\hline Thréonine ......... & 3,75 & 3,7 & Isoleucine $\ldots \ldots \ldots$ & 3,55 & 3,4 \\
\hline Sérine $\ldots \ldots \ldots \ldots$ & 4,8 & 4,3 & Leucine $\ldots . . \ldots \ldots$ & 13,3 & 8,9 \\
\hline Acide glutamique ... & 20,0 & 16,1 & Tyrosine $\ldots .$. & 4,6 & 3,6 \\
\hline Proline...$\ldots \ldots$. & 10,2 & 8,55 & Phénylalanine. & 5,1 & 4,45 \\
\hline Glycine $\ldots \ldots \ldots \ldots$ & 3,65 & 4,45 & Lysine . . . . . & 2,95 & $t_{4}, 8$ \\
\hline Alanine $\ldots . .$. & 7,6 & 6,2 & Histidine ...... & 2,9 & 3,3 \\
\hline Cystine...$\ldots \ldots$ & $2, \mathbf{t}^{\prime}$ & 2,75 & Arginine .. & 4,65 & 6,95 \\
\hline Méthionine ........ & 2,25 & 1,6 & MA p. 100 MS .. & 11,30 & 11,53 \\
\hline
\end{tabular}

Dosages effectués par Jeanine Jung (1971), Station de Recherches sur l'élevage des Porcs.

(tous les I 4 jours). A $60 \mathrm{~kg}, 4$ animaux ( 2 mâles castrés et 2 femelles) sont abattus dans chaque lot. Les autres sont sacrifiés à $90 \mathrm{~kg}$. Dans les deux cas, les carcasses sont découpées selon la technique parisienne, après un ressuyage de 24 heures. On procède en outre à la mesure du poids apparent du jambon, de l'ensemble longe + bardière et de la poitrine, après immersion dans l'eau à une température constante $\left(7^{-8}{ }^{\circ} \mathrm{C}\right)$, suivant la technique décrite par Desmoulin (I970).

\section{II. - RÉSUL'TA'TS}

\section{A. - Croissance et consommation (tabl. 3)}

La comparaison statistique des traitements ne fait apparaître globalement aucune différence significative dans les performances réalisées au cours de la première phase de la croissance (entre 29 et $60 \mathrm{~kg}$ de poids vif). Il est à noter cependant que le régime témoin $\mathrm{I}$, à base de maïs normal, semble se distinguer de ceux à base de maïs Opaque 2 par une vitesse de croissance supérieute, et surtout par un indice de consommation plus faible $(3,13$ contre $3,39-3,47$ et 3,39 , respectivement dans les lots 2,3 et 4), pour une consommation moyenne journalière d'aliment sensiblement égale. Ces différences sont encore plus accentuées si l'on exprime l'indice de consommation en quantité de matière sèche par $\mathrm{kg}$ de gain, compte tenu des écarts de teneur en matière sèche entre les régimes Opaque $(89,5 \mathrm{p}$. I0o) et le régime témoin $(87,4 \mathrm{p}$. I00).

Par contre, pendant la période de finition (entre 60 et $90 \mathrm{~kg}$ de poids vif), les résultats sont peu différents d'un lot à l'autre. Tout au plus peut-on remarquer une légère augmentation de l'indice de consommation chez les animaux du lot 3 (maîs Opaque 2 à taux réduit de soja + lysine), soit 3,89 contre 3,6I-3,65 et 3,59 respectivement dans les lots I, 2 et 4 .

Sur l'ensemble de l'expérience, la durée plus longue de la période d'observation permet de conclure d'une manière significative (seuil 0,05 ) à la supériorité du gain 
UTILISATION DU MAÏS “ OPAQUE 2 "PAR LE PORC

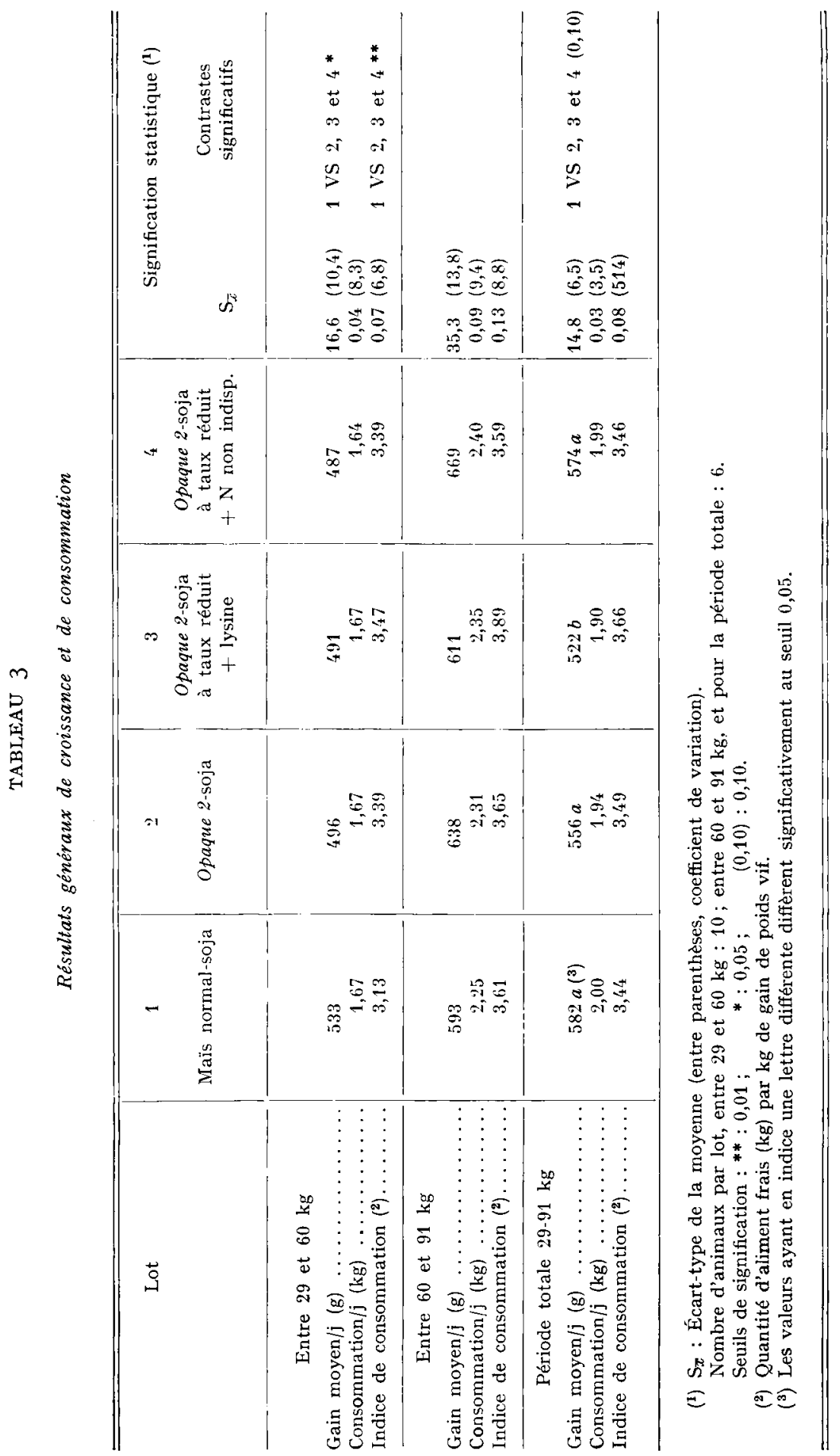




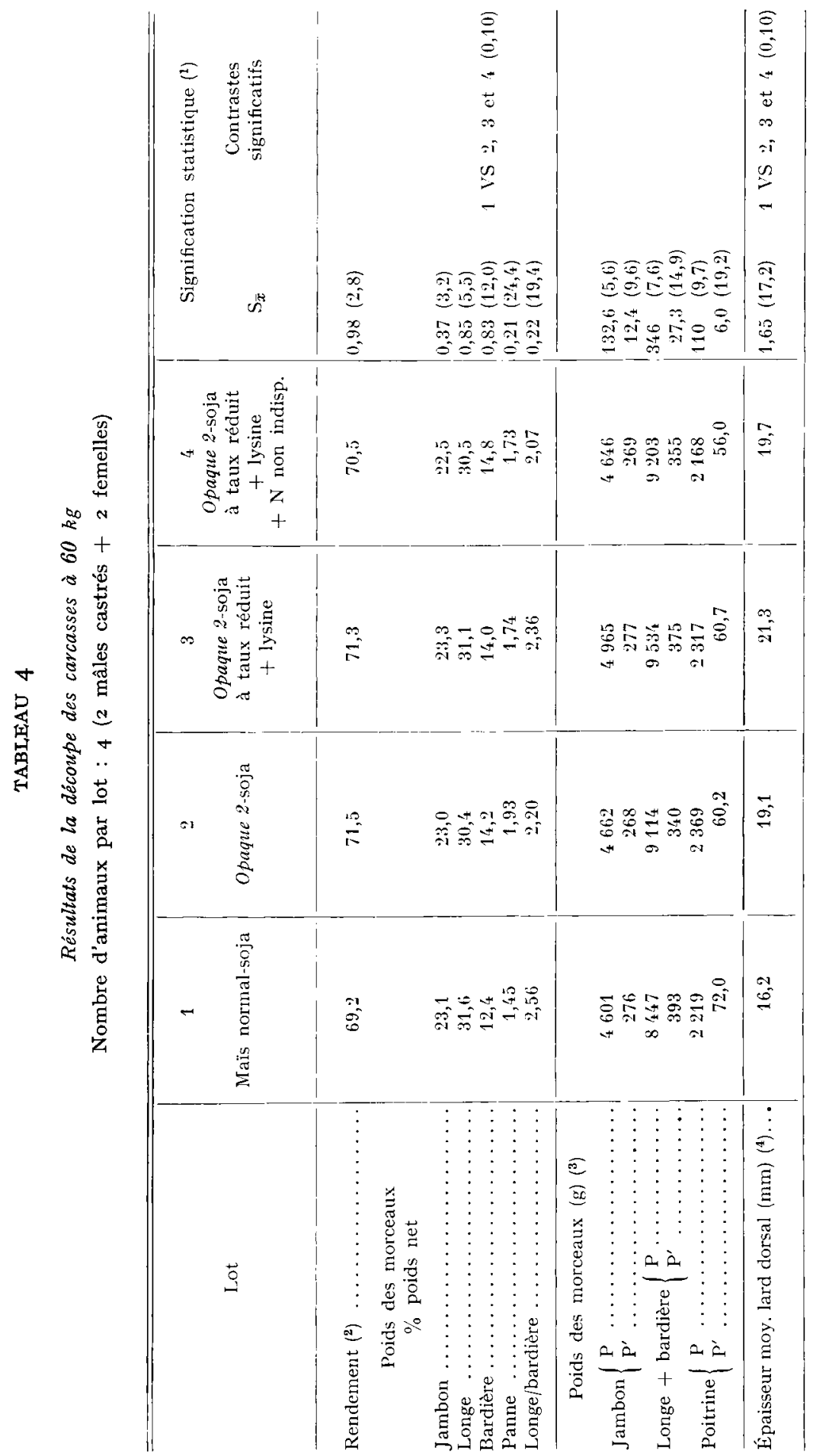


moyen journalier dans les lots I, 2 et 4 sur celui réalisé par le lot 3 (mais Opaque 2 à taux réduit de soja) : soit 582,556 et $574 \mathrm{~g} /$ jour dans les lots $\mathrm{I}, 2$ et 4 contre 522 dans le lot 3. De la même façon, 1'indice de consommation est plus élevé dans ce dernier lot : 3,66 contre 3,44-3,49 et 3,46 dans les lots I, 2 et 4 .

\section{B. - Composition corporelle}

a) $A 60 \mathrm{~kg}$.

Compte tenu de la variabilité des critères et du faible nombre d'animaux observés (4 par lot), on ne note aucune différence significative entre les 4 lots. Remarquons toutefois que le rendement à l'abattage a tendance être plus faible chez les animaux nourris au maîs " normal " (lot I) que chez ceux des lots Opaque 2 (2,3 et 4). Il en est de même pour les critères d'adiposité : pourcentage de bardière et de panne, rapport

\section{TABLEAU 5}

Résultats de la découpe des carcasses à $91 \mathrm{~kg}$

Nombre d'animaux par lot : 6 (3 mâles castrés +3 femelles)

\begin{tabular}{|c|c|c|c|c|c|}
\hline Lot & $\begin{array}{l}\text { Maīs normal- } \\
\text { soja }\end{array}$ & $\begin{array}{l}\text { Opaque 2- } \\
\text { soja }\end{array}$ & $\begin{array}{l}\text { Opaque 2-soja } \\
\text { à taux réduit } \\
+ \text { lysine }\end{array}$ & $\begin{array}{c}\text { Opaque } 2 \text {-soja } \\
\text { à taux réduit } \\
+ \text { lysine } \\
+\mathrm{N} \text { non indisp. }\end{array}$ & $\begin{array}{c}\text { Signification } \\
\text { statistique }\left({ }^{(1)}\right. \\
\mathrm{S}_{\bar{x}}\end{array}$ \\
\hline Rendement $\left({ }^{2}\right)$ & 73,5 & 73,5 & 74,5 & 73,7 & $0,61 \quad(2,0)$ \\
\hline $\begin{array}{l}\text { Poids des morceaux } \\
\% \text { poids net }\end{array}$ & & & & & \\
\hline Jambon $\ldots \ldots \ldots \ldots$ & 21,3 & 21,1 & 21,6 & 21,0 & $0,33(3,8)$ \\
\hline Longe $\ldots \ldots \ldots \ldots$ & 30,4 & 30,2 & 29,5 & 29,7 & $0,43(3,6)$ \\
\hline Bardière $\ldots \ldots \ldots \ldots$. & 16,7 & 16,3 & 16,7 & 16,2 & $0,5^{\prime} \mathbf{t}(8,0)$ \\
\hline Panne............ & 2,53 & 2,96 & 2,73 & 2,88 & $0,17(15,2)$ \\
\hline Longe/bardière & 1,84 & 1,87 & 1,79 & 1,86 & $0,08(11,2)$ \\
\hline $\begin{array}{l}\text { Poids des morceaux } \\
\text { (g) }{ }^{(3)}\end{array}$ & & & & & 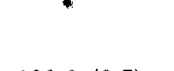 \\
\hline Jambon $\quad\left\{\begin{array}{l}\mathrm{P} \ldots \\
\mathrm{P}^{\prime} \ldots\end{array}\right.$ & $\begin{array}{r}6968 \\
373\end{array}$ & $\begin{array}{r}7023 \\
360\end{array}$ & $\begin{array}{r}7196 \\
352\end{array}$ & $\begin{aligned} 6980 \\
340\end{aligned}$ & $130,6 \quad(3,7)$ \\
\hline Longe $\quad$ P .... & 15670 & 15547 & 15520 & $\begin{array}{r}340 \\
15435\end{array}$ & $\begin{aligned} 13,6 & (8,8) \\
235,7 & (3,0)\end{aligned}$ \\
\hline + Bardière $\mid \mathrm{P}^{\prime} . .$. & 518 & $50^{\prime}$ & 466 & 468 & $43,3(17,7)$ \\
\hline Poitrine $\quad\left\{\begin{array}{l}\mathrm{P} \\
\mathrm{P}\end{array}\right.$ & 3510 & 3447 & 3459 & 3703 & $86,1 \quad(4,9)$ \\
\hline $\mid \mathrm{P}^{\prime} \ldots$ & 65,7 & 60,5 & 40,7 & 63,0 & $11,6 \quad(40,3)$ \\
\hline $\begin{array}{c}\text { Épaisseur moyenne du } \\
\text { lard dorsal }(\mathrm{m} / \mathrm{m})\end{array}$ & 25,3 & 24,8 & 25,5 & 24,8 & $1,4 \quad(13,8)$ \\
\hline
\end{tabular}

(1) $\mathrm{S}_{\not{x}}$ : Écart type de la moyenne (6 animaux); entre parenthèses, coefficient de variation.

(2) Poids net, sans la tête et avec les pieds, après un ressuyage de 24 heures (p. 100 poids vif).

( $\left.{ }^{3}\right) \mathrm{P}$ : Poids brut déterminé dans l'air. $\mathrm{P}^{\prime}$ : Poids apparent déterminé après immersion du morceau dans l'eau par densimétrie.

Les résultats portent seulement sur 4 animaux par lot ( 2 mâles castrés et 2 femelles).

(4) $\frac{\text { Rein }+ \text { dos }}{2}$. 
longe/bardière, épaisseur moyenne du lard dorsal. Cette tendance à une adiposité réduite des carcasses du lot I semble être confirmée par l'évolution des poids apparents de 1'ensemble (longe + bardière) et de la poitrine, dont l'augmentation traduit une proportion plus importante de masses maigres. Ces résultats sont à rapprocher de la diminution de l'indice de consommation déjà signalée dans le même lot, au cours de la première phase de la croissance (tabl. 4).

b) $A 91 \mathrm{~kg}$.

A la fin de l'expérience, les traitements sont sans effet sur les résultats de la découpe des carcasses, qu'il s'agisse du rendement, des poids des morceaux relativement au poids net, de leurs poids apparents ou de l'épaisseur moyenne du lard dorsal (tabl. 5).

\section{III. - DISCUSSION ETT CONCLUSION}

Les résultats de la présente étude ont montré qu'à taux de matières azotées et de lysine comparables, le régime contenant du maïs normal procure pendant la première phase de la croissance des performances supérieures à celles obtenues avec les régimes à base de maîs Opaque 2 , tandis que les carcasses des animaux abattus à $60 \mathrm{~kg}$ présentent une adiposité moindre. Pour expliquer cette différence, on pourrait penser à l'existence d'un déficit en un ou plusieurs acides aminés indispensables dans les régimes renfermant le maîs Opaque 2. Cette éventualité paraît cependant exclue. La teneur en tryptophane, qui constitue l'acide aminé limitant de ces régimes, est en effet sensiblement la même que dans le régime témoin, soit o,I $6 \mathrm{p}$. Ioo, et correspond à la dose habituellement recommandée pour le porc en croissance (N. R. C., I g68). Il en est de même pour la thréonine, dont les teneurs dans les protéines du maïs et du soja sont assez peu différentes, soit respectivement 3,7 et 4 , I g pour I6 $\mathrm{g}$ d'N (d'après Pion, I97I). Malgré les résultats favorables obtenus sur le rat par VERMOREL, (I969, I97I), avec le maîs Opaque 2, on pourrait émettre l'hypothèse d'une disponibilité plus faible des acides aminés dans les protéines de maïs que dans celles de soja, qui reprêsentent respectivement 55 et $45 \mathrm{p}$. Ioo des protéines totales de la ration, suivant que cette dernière contient du maïs normal ou du mais Opaque 2.

Quoi qu'il en soit, si l'on considère les performances enregistrées sur l'ensemble de la croissance, les régimes à base de maïs Opaque 2 se révèlent comparables au régime témoin renfermant du maïs normal, à la condition, cependant, que non seulement la teneur en lysine soit la même, mais également celle des matières azotées, à la suite des résultats inférieurs observés dans le lot 3 à faible taux de matières azotées (r3 p. I0o) pendant la période de finition.

En définitive, la valorisation complète de la lysine du maïs Opaque 2, en association avec le tourteau de soja seul, risque, en abaissant le taux azoté de la ration en dessous d'un seuil critique de retentir défavorablement sur les performances de croissance et la qualité des carcasses. L'économie de $5 \mathrm{p}$. Ioo de tourteau de soja, déjà signalée précédemment, n'est possible en réalité que si elle est compensée par un apport complémentaire d'azote non indispensable. Si cela n'était pas le cas, il faudrait que la teneur en matières azotées du maïs Opaque 2 soit accrue de 5 p. Ioo environ, c'est-à-dire que l'on puisse disposer d'un maîs à la fois riche en lysine et à teneur 
plus élevée en matières azotées. Tant que le tourteau de soja constituera le complément azoté principal dans l'alimentation du porc, on peut s'interroger sur l'intérêt d'un maïs simplement mieux pourvu en lysine que le maïs normal. Seule l'extension de nouvelles sources azotées riches, mais relativement déficientes en lysine, comme le tourteau de tournesol, pourrait laisser espérer une meilleure valorisation du maïs Opaque 2.

Reçu pour publication en janvier 1973.

\author{
SUMMARY
}

UTILIZATION OF " OPAQUE 2 " MAIZE BY GROWING-FINISHING PIGS

The aim of the present experiment carricd out with 40 Large White pigs between 29 and $90 \mathrm{~kg}$ live weight was to examine the utilization conditions of "Opaque 2 " maize in comparison with normal maize in diets supplemented by soybean oil-meal.

Four groups of to animals each received the following diets according to the full hand feeding method :

- Group I - Control diet - normal maize soybean oil-meal (I6.5 p. too crude protein and $0.8 \mathrm{p}$. Ioo lysine during the growth period, $14.5 \mathrm{p}$. I 00 crude protein and $0.65 \mathrm{p}$. roo lysine during the finishing period).

- Groupe 2 - "Opaque 2 " maize - soybean oil-meal diet with the same supply of crude protein and lysine as the control diet.

- Group 3 - "Opaque 2 " maize diet with reduced soybean level (14.5 p. Ioo crude protein during the growth period and I 2.5 during the finishing period) and supplemented by lysine.

- Group 4 - Diet 3 rebalanced at the same crude protein level as the control diet (Group I), with supplementary supply of maize gluten.

The performances obtained during this experiment were almost the same for the two types of maize, except, however, for Group 3 (reduced $\mathrm{N}$ level) which appeared to be inferior to the three others. In addition, during the first phase of fattening, the animals receiving the diets based on "Opaque 2 " maize showed slightly poorer growth performances than those of the controls (on an average 49I $\mathrm{g}$ for «Opaque 2 " maize groups versus $533 \mathrm{~g}$ for the normal maize group).

\title{
RÉFÉRENCES BIBLIOGRAPHIQUES
}

Cromwell G. L., Pickett R. A., Beeson W. M., ig67. Nutritional value of Opaque 2 corn for swine. J. Anim. Sci., 26, I325-I33I.

Desmovlin 13., I97o. La détermination de la densité corporelle : principes et conditions d'une mesure directe de l'état d'engraissement de la carcasse du Porc. J. Rech. Porcine en France, I. N. R. A., Paris, I. T. P. Ed., I 7 I-I75.

Drews J. E., Moody N. W., Hays V. W., Speer V. C., Ewan R. C., Ig69. Nutritional value of Opaque 2 corn for young chicks and pigs. J. Nutr., 97, 537-54I.

Gallo J. T., Maner J. H., Corzo M., r969. Nutritive value of Opaque 2 corn for the finishing pigs. J. Anim. Sci., 29, 134 (Abstr.).

Gipp IV. C., Cline T. R., Rogler J. C., I968. Nutritional studies with opaque 2 and high protein Opaque 2 corn. J. Anim. Sci., 27, r775 (Abstr.).

HeNRY Y., I972. Effets comparés de la lysine et des matières azotées sur les performances du porc en finition. J. Rech. Porcine en France, I. N. R. A., Paris, I. 'T. P. Éd., I63-168.

Henry Y., Bourdon D., I97r. Utilisation de nouveaux types de céréales dans l'alimentation du Porc en croissance-finition : avoine nue, maïs Opaque 2. J. Rech. Porcine en France, I. N. R. A., Paris, I. T. P. Ed., I53-159.

N. R. C., r968. Nutrient requirements for swine. Publ. I599. National Academy of Science. National Research Council., Washington D. C. 
Pion R., I97r. Composition en acides aminés des aliments. Ind. Alim. Anim., 6, 29-36.

Sinombing D. T. H., CRomwell G. L., HAYS V. W., I969. Growth and digestibility studies with Opaque 2 and normal corn for growing pigs. J. Anim. Sci., 29, $921-926$.

Vermorel M., I969. Utilisation énergétique par le Rat en croissance d'un maïs hybride Opaque 2 composant un régime équilibré en acides aminés. C. $R$. Acad. Sci., Paris, 268, 834-837.

VERMOREL M., I97I. Utilisation énergétique et azotée de quelques aliments pour les monogastriques en croissance. Études sur le Rat. X' Congrès International de Zootechnie, thème VII, Nutrition, Versailles. 